\title{
Chiral behavior of pseudo-Goldstone boson masses and decay constants in $2+1$ flavor QCD
}

\author{
S. Dürr ${ }^{1}$, Z. Fodor ${ }^{1,2,3}$, C. Hoelbling ${ }^{2,5}$, S.D. Katz ${ }^{2,3}$, S. Krieg ${ }^{2,4}$, Th. Kurth ${ }^{2}$, \\ L. Lellouch ${ }^{* 5}$, Th. Lippert ${ }^{1,2,4}$, K.K. Szabo ${ }^{2}$, G. Vulvert ${ }^{5}$ \\ ${ }^{1}$ John von Neumann Institute for Computing (NIC), DESY, D-15738, Zeuthen / FZJ, D-52425, \\ Juelich, Germany \\ ${ }^{2}$ Department of Physics, University of Wuppertal, D-42097 Wuppertal, Germany \\ ${ }^{3}$ Institute for Theoretical Physics, Eötvös University, H-1117 Budapest, Hungary \\ ${ }^{4}$ Jülich Supercomputing Center (JSC), Forschungszentrum Jülich, D-52425 Jülich, Germany \\ ${ }^{5}$ Centre de Physique Théorique, Case 907, Campus de Luminy, F-13288 Marseille Cedex 9, \\ France $^{\dagger}$
}

\section{E-mail: lellouchecpt.univ-mrs.fr}

\begin{abstract}
We present preliminary results for the chiral behavior of charged pseudo-Goldstone-boson masses and decay constants. These are obtained in simulations with $N_{f}=2+1$ flavors of tree-level, $O(a)$ improved Wilson sea quarks. In these simulations, mesons are composed of either valence quarks discretized in the same way as the sea quarks (unitary simulations) or of overlap valence quarks (mixed-action simulations). We find that the chiral behavior of the pseudoscalar meson masses in the mixed-action calculations cannot be explained with continuum, partially-quenched chiral perturbation theory. We show that the inclusion of $O\left(a^{2}\right)$ unitarity violations in the chiral expansion resolves this discrepancy and that the size of the unitarity violations required are consistent with those which we observe in the zero-momentum, scalar-isotriplet-meson propagator.
\end{abstract}

The XXV International Symposium on Lattice Field Theory

July 30 - August 42007

Regensburg, Germany

\footnotetext{
* Speaker.

${ }^{\dagger}$ CPT is "UMR 6207 du CNRS et des universités d'Aix-Marseille I, d'Aix-Marseille II et du Sud Toulon-Var, affiliée à la FRUMAM.”
} 


\section{Introduction}

The objective of our collaboration is to calculate hadronic observables which are relevant for determining fundamental quark properties, such as quark masses or quark flavor-mixing and CPviolation parameters, and to do so with controlled extrapolations to the physical limit of $N_{f}=2+1$ flavor QCD, where $M_{\pi} \simeq 135 \mathrm{MeV}$, the lattice spacing $a$ vanishes and the volume is infinite. To achieve that goal we consider two approaches. In both, the seas are composed of $N_{f}=2+1$ flavors of tree-level, $O(a)$-improved Wilson (W) quarks [1]. We perform "unitary" simulations where the valence quarks are discretized in the same way as the sea quarks. We also perform "mixed-action" calculations, with overlap [2-包, Ginsparg-Wilson (GW) [5] valence quarks. In the latter, the valence sector possesses a full, continuum-like chiral symmetry [6] which greatly simplifies the renormalization of electroweak operators, such as those encountered in neutral kaon mixing. It also guarantees that matrix elements are automatically $O(a)$-improved, to the extent that the sea quarks are. As discussed by Stefan Krieg at this conference [7], recent advances have allowed us to perform $N_{f}=2+1$ simulations, for instance, down to $M_{\pi} \sim 190 \mathrm{MeV}$ with $a \sim 0.09 \mathrm{fm}$ and in cubic volumes of side $L \sim 4.2 \mathrm{fm}$. Thus, we expect to be able to reach the near-continuum chiral $p$-regime of Gasser and Leutwyler without the conceptual problems of staggered fermions [8, 9]. This means that we should be able to extrapolate lattice results to the physical point in a modelindependent way, by using Wilson [10-13], partially quenched (PQ) chiral perturbation theory $(\chi \mathrm{PT})$ [14- 17] for the unitary simulations, and mixed-action (MA) PQ $\chi$ PT [18, 13, 19] for the GW-on-W simulations.

One of the drawbacks of using a mixed-action approach is the presence of discretizationinduced unitarity violations. Fortunately, it should be possible to account for the low-energy manifestations of these violations with MAPQ $\chi \mathrm{PT}$. We present here preliminary results for the quarkmass dependence of the masses and leptonic decay constants of the pseudo-Goldstone bosons (PGBs) of chiral symmetry breaking. In particular, we investigate the effects of unitarity violations in these quantities, as obtained in our mixed-action simulations, and attempt to correlate these effects with those which we observe in the scalar, isotriplet, $a_{0}$ propagator, where they are expected to be particularly large [20]. Of course, our study of the masses and decay constants of the PGBs is primarily motivated by the very interesting phenomenology they give rise to. They allow the determination of a variety of fundamental quantities, such as light quark masses, the ratio of CKM elements $\left|V_{u s} / V_{u d}\right|$ [21] and important LO and higher-order low-energy constants (LECs) of the effective chiral Lagrangian. However, we postpone the presentation of results for these quantities to later publications.

\section{Finite-volume mixed-action $P Q \chi P T$ and unitarity violations}

As shown in [20] for the case of $N_{f}$ degenerate flavors, the propagator of the $a_{0}$ is affected by potentially large $O\left(a^{2}\right)$ unitarity violations in a mixed-action scenario. For the case which interests us, with $N_{f}=2+1$, to simplify expressions we suppose that the light sea $(\ell)$ and valence $(v)$ quark masses are tuned such that masses of the corresponding PGBs are equal: $M_{v v}=M_{\ell \ell} \equiv M_{\pi}$. We denote the strange sea quark by $s$, and by $M_{s s}$ the mass of charge pseudoscalar mesons composed of two valence quarks with the mass of the $s .{ }^{1}$ Then, LO MAPQ $\chi$ PT gives for the zero-momentum,

\footnotetext{
${ }^{1}$ From now on we call these mesons "non-singlet $s \vec{s}$ " pseudoscalar mesons.
} 
$a_{0}$ propagator:

$$
\begin{aligned}
C_{a_{0}}(t, \lambda) & \equiv Z_{S}^{2}\left(g_{0}, a \lambda\right) a^{3} \sum_{\vec{x}}\left\langle\bar{q}_{2} q_{1}(\vec{x}, t) \bar{q}_{1} q_{2}(0)\right\rangle \\
& \stackrel{t \rightarrow+\infty}{\longrightarrow} \frac{B^{2}(\lambda)}{L^{3}}\left\{C_{K \bar{K}}(t)+\frac{2}{3} C_{\pi \eta}(t)-2 \frac{a^{2} \Delta}{M_{\pi}^{2}}\left(M_{\pi} t+1\right) C_{\pi \pi}(t)\right\},
\end{aligned}
$$

where the valence quarks are chosen to be degenerate, with $m_{1}=m_{2} \doteq m_{v}$, and where we have assumed, for simplicity, that the time and space extent of the lattice are infinite. The quantity $B$ is the condensate over the square of the decay constant $F . Z_{S}\left(g_{0}, a \lambda\right)$ is a renormalization constant for scalar densities and $\lambda$ is a QCD renormalization scale. The functions $C_{X Y}(t)$ denote the zeromomentum propagators of the two-particle states, $X Y$, and are given by $C_{X Y}(t) \equiv \exp \left[-\left(M_{X}+\right.\right.$ $\left.\left.M_{Y}\right) t\right] /\left(4 M_{X} M_{Y}\right)$ at LO. There are two physical contributions, coming from intermediate two-kaon and $\pi \eta$ states. The unitarity violations are of order $a^{2}$ and are proportional to a quantity $\Delta$, which has mass dimension four. These violations only vanish in the continuum limit. Moreover, they are exponentially and polynomially enhanced in $t$, also compared to the contribution from the $a_{0}$ not shown in Eq. (2.1). At asymptotic times, the unitarity violations are the dominant contribution.

Unitarity violations also affect PGB masses and decay constants, but only at NLO. Let us consider a pseudoscalar meson composed of two distinct quarks with masses $m_{1}$ and $m_{2}$. Then, according to NLO MAPQ $\chi$ PT, the square of this meson mass has the following generic form:

$$
\begin{aligned}
\left(M_{12}^{2}\right)_{\Omega}^{\mathrm{NLO}}=\left(m_{1}+m_{2}\right) & B\left\{1+\frac{1}{(4 \pi F)^{2}}\left[\mathrm{PQ}-\operatorname{logs}\left(\mu, M_{11}, M_{22}, M_{\ell \ell}, M_{s s}\right)\right.\right. \\
+ & \left(2 \alpha_{6}-\alpha_{4}\right)(\mu)\left(2 M_{\ell \ell}^{2}+M_{s s}^{2}\right)+\left(2 \alpha_{8}-\alpha_{5}\right)(\mu) M_{12}^{2}+\mathrm{FV} \\
+ & \left.\left.a \beta_{M}+a^{2} \Delta \times\left\{\mathrm{UV}-\operatorname{logs}\left(\mu, M_{11}, M_{22}\right)+\gamma_{M}(\mu)\right\}\right]\right\},
\end{aligned}
$$

where $B$ and $\Delta$ are defined after Eq. (2.1); "PQ-logs" and "UV-logs" denote partially-quenched and unitarity-violating quenched-like logarithms, respectively; $\mu$ is the renormalization scale in the effective theory and, in Eq. (2.2), the quark masses and the $B$ must both be either renormalized in the same scheme in QCD or bare; $\gamma_{M}(\mu)$ is an a priori unknown counter-term; the LECs $\alpha_{i}$ are related to the original Gasser and Leutwyler constants through $\alpha_{i}(\mu) \equiv 8(4 \pi)^{2} L_{i}(\mu)$; FV stands for finite-volume corrections; $\beta_{M}$ is a mass-dimension three quantity which parameterizes $O(a)$ discretization errors and whose parametric size will be specified below. Note that in fits to lattice results obtained at a single lattice spacing, the discretization errors proportional to $a \beta_{M}$ and $a^{2} \Delta \gamma_{M}(\mu)$ get absorbed into the LO LEC, $B$.

In applying the general form of Eq. (2.2), it is useful to distinguish three cases of interest. In the continuum or in the case of GW valence on GW sea quarks, $m_{1}$ and $m_{2}$ can be taken to be the Lagrangian masses and $\Delta$ and $\beta_{M}$ identically vanish, i.e. there are no unitarity violating nor $O(a)$ discretization errors. In our W-on-W unitary simulations, we take $m_{1}$ and $m_{2}$ to be the "measured" axial Ward identity (AWI) masses. Moreover, the constant $\beta_{M}$ is $O\left(\Lambda_{\mathrm{QCD}}^{3}\right)$ if the fermions used are straight Wilson fermions, $O\left(\alpha_{s} \Lambda_{\mathrm{QCD}}^{3}\right)$ if they are tree-level $O(a)$-improved as they are in our simulations, or zero if they are non-perturbatively $O(a)$-improved. Finally, $\Delta \equiv 0$ and discretization-induced unitarity violations are absent. In the mixed-action, GW-on-W case, $m_{1}$ and $m_{2}$ can be taken to be the valence, overlap Lagrangian masses. $\beta_{M}$ has the same parametric size as for the unitary simulations, depending on the level of improvement of the sea. However, in the mixed-action case, $\Delta$ does not vanish a priori. Thus, we expect that meson masses will suffer from discretization-induced unitarity violations at finite $a$. 
The NLO expression for the decay constant, $F_{12}$, of a charged pseudoscalar meson is similar in form to Eq. (2.2), with the factor $\left(m_{1}+m_{2}\right) B$ replaced by $F$ and where the "PQ-logs"and corresponding counter-terms and finite-volume corrections are changed to the partially-quenched expressions appropriate for decay constants. The corresponding general form applies to the three cases discussed above in much the same way, with the substitutions $\beta_{M} \rightarrow \beta_{F}$ and $\gamma_{M} \rightarrow \gamma_{F}$. The main difference appears in the GW-on-W case, where the mixed-action unitarity violations proportional to $a^{2} \Delta$ are purely valence $S U(3)$-flavor breaking, and do not depend on $\mu$.

\section{Results for the $a_{0}$ propagator and the charged PGB masses and decay constants}

The results discussed below were obtained from simulations which were described at this conference by Stefan Krieg [7]. We recapitulate here only the main ingredients, omitting algorithmic considerations. The gauge action used is tree-level Symanzik improved [22]. The sea quarks are described by six-step stout-smeared [23], tree-level $O(a)$-improved Wilson fermions [1]. For the unitary, W-on-W simulations, the valence quarks are discretized in the same way. In the GW-on-W, mixed-action case, valence quarks are three-step HYP-smeared [24] overlap fermions [2]-印, with a negative mass parameter $\rho=1$.

We have performed five, $2+1$ sea-flavor simulations at a lattice spacing $a \sim 0.09 \mathrm{fm}$ ( $\beta=$ 3.57). In these simulations, the mass of the charged pions composed of two light sea quarks are $M_{\pi} \sim 190,300,410,490$ and $570 \mathrm{MeV}$. To keep finite-volume errors small, all simulations are performed in cubic three-volumes with sides $L$ such that $M_{\pi} L \geq 4$. The strange quark mass used in our simulations is slightly overestimated: with such a strange quark, the mass of a kaon, when extrapolated in light-quark mass to the physical point, is approximately $7 \%$ higher than the physical kaon mass. There are 34 gauge configurations at $M_{\pi} \sim 190 \mathrm{MeV}, 68$ at $M_{\pi} \sim 300 \mathrm{MeV}$ and $O(100)$ at the three other simulation points. In the mixed-action case, the overlap quark masses are chosen such that the mesons which they compose are approximately degenerate with those composed of the corresponding Wilson sea quarks. At $M_{\pi} \sim 190$ and $300 \mathrm{MeV}$, we have a second overlap valence strange quark whose mass is approximately $30 \%$ smaller than that of the strange sea quark.

We begin with preliminary results for the zero-momentum, $a_{0}$ propagator, $C_{a_{0}}(t)$, defined in Eq. (2.1). In Fig. 11 we plot the unrenormalized propagators, $C_{a_{0}}^{\text {bare }}(t)$, as a function of Euclidean time $t$, obtained in the two GW-on-W simulations with the lightest $u$ and $d$ quarks. The propagators go negative at relatively short times and then asymptotically go back up to zero. Moreover, the effect is less pronounced for the simulation with the more massive quarks, a trend which persists as one increases the $u$ and $d$ quark masses further. This behavior is qualitatively consistent with the prediction of MAPQ $\chi \mathrm{PT}$ given in Eq. (2.1), assuming $\Delta>0$. The agreement can be made quantitative also. To verify this, we perform fits of the propagators, at asymptotic times $t$, to the PQ and FV generalization of Eq. (2.1) for the bare $C_{a_{0}}(t)$. The fits have only one parameter, namely $a^{4} \Delta$. For the pre-factor $\left(B / Z_{S}\right)^{2}$, we take $M_{12}^{2} /\left(m_{1}+m_{2}\right)$, where $M_{12}$ is the "measured" meson mass and $m_{1,2}$ are the bare masses of the GW quarks which compose it. The kaon, pion and $\eta$ masses which appear in the expression for $C_{a_{0}}^{\text {bare }}(t)$ are constrained to take on the values obtained from prior fits to kaon and pion two-point functions. These mesons are composed of a sea and a valence quark. We obtain their masses by combining the corresponding valence-valence and sea-sea masses at LO in the chiral expansion. This means that the parameter $a^{4} \Delta$ that we fit here contains contributions from an $O\left(a^{2}\right)$, mixed-action operator in the chiral Lagrangian. Fortunately, 


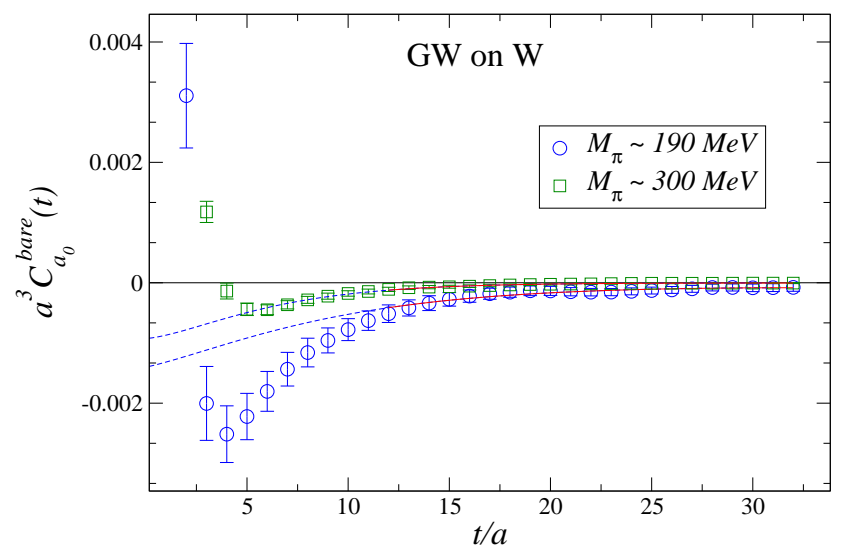

Figure 1: Bare, zero-momentum propagator of the $a_{0}$ as a function of time over half the time extent of our lattices, as obtained in our two GW-on-W simulations with the lightest $u$ and $d$ quarks. The solid curves represent our best fits to the partially-quenched and finite-volume generalization of Eq. (2.1) in the fit region, and the dashed curves their extensions to earlier times.

if the same prescription is used for obtaining valence-sea meson masses which appear at NLO in quantities such as $M_{12}^{2}$ or $F_{12}$, the unitarity violations there will be parameterized by the same $a^{4} \Delta$.

The one-parameter fits to $C_{a_{0}}^{\text {bare }}(t)$ for $M_{\pi} \sim 190$ and $300 \mathrm{MeV}$ are performed for $t / a$ in the range $[12,32]$, where $t / a=32$ is the midpoint of our lattices in both cases. The results of these fits are plotted in Fig. 1. As the figure suggests, both fits have good $\chi^{2} / d o f$. The values obtained for the unitarity-violation parameter are $a^{4} \Delta=0.015(6)$ and $0.024(10)$, respectively, for $M_{\pi} \sim 190$ and $300 \mathrm{GeV}$, and are thus consistent. For the lattice spacing at which the simulations are performed, these values correspond to $a \sqrt{\Delta} \sim 0.27 \mathrm{GeV}$ and $0.35 \mathrm{GeV}$. Since $a \sqrt{\Delta}$ competes with pion, kaon and $\eta$ masses in the chiral expressions for $M_{12}^{2}$ and $F_{12}$ in the mixed-action case, it is clear that these unitarity-violating contributions cannot be neglected a priori.

We now turn to an analysis of the GW-on-W decay constant. We begin with this quantity, because we use the extrapolated $a F_{\pi}$ to determine the lattice spacing as well as to normalize corrections in chiral expressions with factors of $\left(4 \pi a F_{\pi}\right)^{2} . a F_{12}$ is obtained from the pseudoscalar two-point function using the AWI. Thanks to the chiral symmetry of the overlap, valence quarks, no renormalization is required. This is a simple example of the simplifications brought about by the use of a mixed action with chirally symmetric valence quarks.

There are 21 lattice points for $a F_{12}$, of which 5 correspond to charged "pions", 7 to "kaons" and 9 to "non-singlet $s \vec{s}$ " pseudoscalar mesons. We fit these results to the NLO chiral expression described in Sec. 2. So as to remain, as much as possible, within in the range of applicability of NLO $\chi \mathrm{PT}$, we include in the fit only the 4 lightest pion and 4 lightest kaon points, with $M_{\pi} \leq$ $500 \mathrm{MeV}$ and $M_{K} \leq 590 \mathrm{MeV}$. The fit has four parameters, which are $F, \alpha_{4}\left(M_{\eta}\right), \alpha_{5}\left(M_{\eta}\right)$ and $a^{4} \Delta$. Since the $a_{0}$ propagator is more sensitive to unitarity violations than are the decay constants, we fix $a^{4} \Delta$ to the value $0.024(10)$, through a Gaussian prior in the $\chi^{2}$. The NLO expression describes the data well. Moreover, the resulting value of $a^{4} \Delta$ is $0.025(8)$, confirming that the chiral behavior of the decay constants is consistent with the presence of unitarity violations of the size observed in the $a_{0}$ propagator. The value of $a F_{\pi}$ obtained from a self-consistent extrapolation to the physical point, using the infinite-volume, continuum fitted function, yields a lattice spacing of $a=0.088(1) \mathrm{fm}$, 

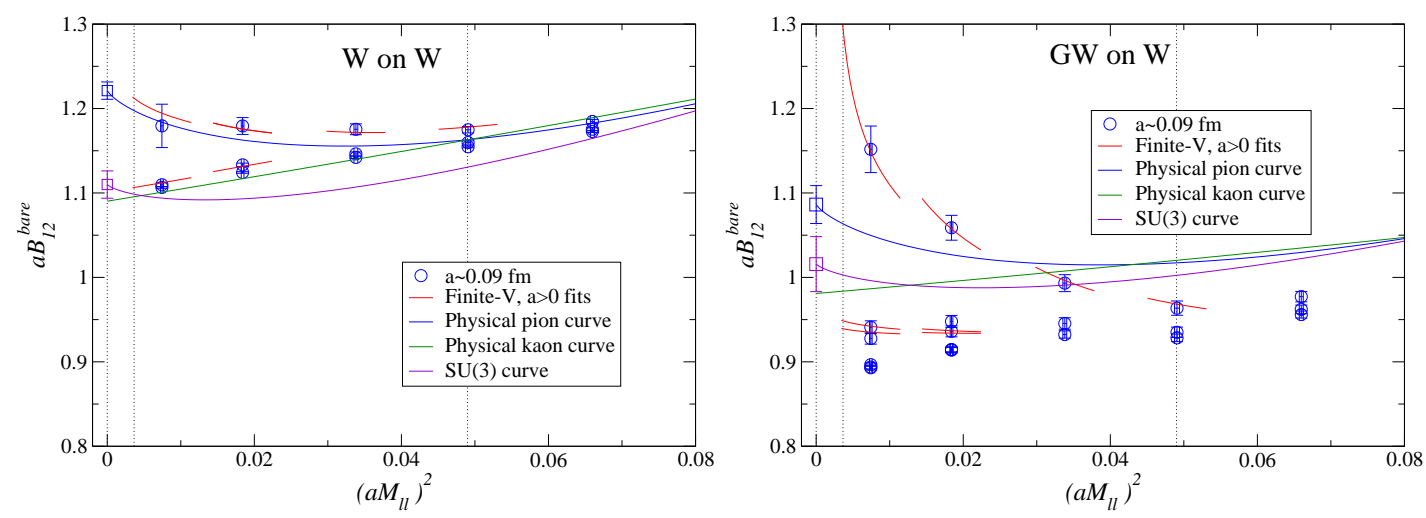

Figure 2: The bare condensate ratio as a function of squared sea-pion mass, in lattice units, for the unitary simulations (left) and for the mixed-action simulations (right). The vertical scales in the two plots are only equal up to a ratio of renormalization constants. The three sets of data (circles) in the W-on-W case correspond, from top to bottom, to pion-like, kaon-like and non-singlet $s \bar{s}$-like pseudoscalar mesons. In the GW-on-W plot, there are one additional kaon and two extra $s \bar{s}$ points at the two smallest $M_{\ell \ell}$ 's, corresponding to the additional valence strange quark that we consider in these simulations. The fits to Eq. (2.2) are plotted as line-segments around each fitted point. The physical curves are obtained from the fits by removing the FV effects, and in the mixed-action case, the partial-quenching effects and unitarity-violating logs. The pion curves are obtained by setting $M_{11}=M_{22}=M_{\ell \ell}$ and $M_{s s}=M_{s S}^{\text {phys }}$, the latter being the physical, non-singlet $s \bar{s}$ pseudoscalar meson mass; the kaon curves, by setting $M_{11}=M_{\ell \ell}$ and $M_{22}=M_{s s}=M_{s s}^{\text {phys }}$; the $S U$ (3) curves, by setting $M_{11}=M_{22}=M_{s s}=M_{\ell \ell}$. The vertical dotted lines mark, from left to right, the chiral limit, the physical pion and the physical kaon points.

where the error is statistical.

Next we consider chiral fits to the unitary W-on-W results for the condensate ratio $a B_{12}^{\text {bare }} \equiv$ $\left(a M_{12}\right)^{2} /\left(a m_{1}+a m_{2}\right)_{\mathrm{AWI}}^{\mathrm{bare}}$. The lattice results for this quantity are plotted in Fig. \& , as a function of the squared sea-pion mass, $M_{\ell \ell}^{2}$. The different sets of points correspond to "pions", "kaons" and "non-singlet $\vec{s} \vec{s}$ " pseudoscalar mesons. We fit these results to the NLO expression of Eq. (2.2) with $\Delta \equiv 0$. We include in the fit only the six points with $M_{\pi} \leq 500 \mathrm{MeV}$ and $M_{K} \leq 590 \mathrm{MeV}$. Here, the fit has only three parameters, namely $B,\left(2 \alpha_{6}-\alpha_{4}\right)\left(M_{\eta}\right)$ and $\left(2 \alpha_{8}-\alpha_{5}\right)\left(M_{\eta}\right)$. The NLO expression describes the chiral behavior of $a B_{12}^{\text {bare }}$ very well.

We now turn to the condensate ratio obtained in the mixed-action, GW-on-W simulations. Here, $a B_{12}^{\text {bare }} \equiv\left(a M_{12}\right)^{2} /\left(a m_{1}+a m_{2}\right)^{\text {bare }}$, where $M_{12}$ is the valence meson mass and $m_{1,2}^{\text {bare }}$ are the corresponding bare overlap Lagrangian masses. The results for this quantity are plotted in Fig. 2, again as a function of $M_{\ell \ell}^{2}$. As the plot indicates, the behavior of $a B_{12}^{\text {bare }}$ here deviates significantly from that obtained in the unitary case. Moreover, some of the features of this behavior, such as the large increase of $a B_{12}^{\text {bare }}$ for the "pion" points at small $M_{\ell \ell}^{2}$, cannot be explained with only continuum PQ chiral logarithms: a divergent term at small $M_{\ell \ell}^{2}$ appears to be required. Fortunately, such a contribution is provided by the unitarity violations discussed in Sec. 2 and exhibited in Eq. (2.2). We thus fit the lattice results to the NLO expression of Eq. (2.2), including the unitarity violating term proportional to $a^{2} \Delta$. As for the decay constant fit, we fix $a^{4} \Delta$ to the value $0.024(10)$, obtained from the $a_{0}$ propagator, through a Gaussian prior in the $\chi^{2}$. There are four parameters in the fit, one more than in the W-on-W case. These are $B,\left(2 \alpha_{6}-\alpha_{4}\right)\left(M_{\eta}\right),\left(2 \alpha_{8}-\alpha_{5}\right)\left(M_{\eta}\right)$ and a constrained $a^{4} \Delta$. Again, only the eight points with $M_{\pi} \leq 500 \mathrm{MeV}$ and $M_{K} \leq 590 \mathrm{MeV}$ are 
included. The description of the condensate ratio given by our NLO chiral expression is good. The value of $a^{4} \Delta$ returned by the fit is 0.020(6). As already noted after Eq. (2.2), in a fit performed at fixed lattice spacing, the discretization error proportional to $\gamma_{M}(\mu)$ gets absorbed into $B$, which thereby acquires a spurious $\chi \mathrm{PT} \mu$-dependence. Of course, this dependence will be eliminated, along with all other discretization errors, when the renormalized values of $B$, obtained at different lattice spacings, are extrapolated to the continuum limit. The value of $\mu$ chosen here is $M_{\eta}$. A lower value will raise the physical curves whereas a larger one will lower them. It is worth noting that this spurious $\mu$-dependence cancels at NLO in ratios such as $m_{s} / m_{u d}$ or $\langle\bar{q} q\rangle_{N_{f}=2} /\langle\bar{q} q\rangle_{N_{f}=3}$. Moreover, it only affects the fitted LECs $\left(2 \alpha_{6}-\alpha_{4}\right)\left(M_{\eta}\right)$ and $\left(2 \alpha_{8}-\alpha_{5}\right)\left(M_{\eta}\right)$ very mildly. We find very good agreement between the GW-on-W and W-on-W results for these quantities.

These observations, together with the other results reported on here, suggest that unitarity violations are present in our mixed-action results, and that we can subtract them with MAPQ $\chi$ PT. Of course, simulations at other lattice spacings are required to confirm this conclusion.

\section{Acknowledgments}

Computations are performed on the BlueGene/L at FZ Jülich and on clusters at the University of Wuppertal and at CPT Marseille. This work is supported in part by EU grant I3HP, OTKA grant AT049652, DFG grant FO 502/1, EU RTN contract MRTN-CT-2006-035482 (FLAVIAnet) and by the CNRS's GDR grant n²921 ("Physique subatomique et calculs sur réseau").

\section{References}

[1] B. Sheikholeslami and R. Wohlert, Nucl. Phys. B259 (1985) 572.

[2] D.B. Kaplan, Phys. Lett. B288 (1992) 342 [hep-lat/9206013].

[3] R. Narayanan and H. Neuberger, Nucl. Phys. B443 (1995) 305 [hep-th/9411108].

[4] H. Neuberger, Phys. Lett. B417 (1998) 141 [hep-lat/9707022].

[5] P.H. Ginsparg and K.G. Wilson, Phys. Rev. D25 (1982) 2649.

[6] M. Lüscher, Phys. Lett. B428 (1998) 342 [hep-lat/9802011].

[7] S. Dürr et al., PoS LAT2007 (2007) 113.

[8] S.R. Sharpe, PoS LAT2006 (2006) 022 [hep-lat/0610094].

[9] M. Creutz, arXiv:0708.1295 [hep-lat].

[10] S.R. Sharpe and R.L. Singleton Jr., Phys. Rev. D58 (1998) 074501 [hep-lat/9804028].

[11] G. Rupak and N. Shoresh, Phys. Rev. D66 (2002) 054503 [hep-lat/0201019].

[12] S. Aoki, Phys. Rev. D68 (2003) 054508 [hep-lat/0306027].

[13] O. Bär, G. Rupak and N. Shoresh, Phys. Rev. D70 (2004) 034508 [hep-lat/0306021].

[14] S.R. Sharpe, Phys. Rev. D46 (1992) 3146 [hep-lat/9205020].

[15] C.W. Bernard and M.F.L. Golterman, Phys. Rev. D46 (1992) 853 [hep-lat/9204007].

[16] C.W. Bernard and M.F.L. Golterman, Phys. Rev. D49 (1994) 486 [hep-lat/9306005].

[17] S.R. Sharpe and N. Shoresh, Phys. Rev. D64 (2001) 114510 [hep-lat/0108003].

[18] O. Bär, G. Rupak and N. Shoresh, Phys. Rev. D67 (2003) 114505 [hep-lat/0210050].

[19] J.W. Chen, D. O'Connell and A. Walker-Loud, arXiv:0706.0035 [hep-lat].

[20] M. Golterman, T. Izubuchi and Y. Shamir, Phys. Rev. D71 (2005) 114508 [hep-lat/0504013].

[21] W.J. Marciano, Phys. Rev. Lett. 93 (2004) 231803 [hep-ph/0402299].

[22] M. Lüscher and P. Weisz, Commun. Math. Phys. 97 (1985) 59.

[23] C. Morningstar and M.J. Peardon, Phys. Rev. D69 (2004) 054501 [hep-lat/0311018].

[24] A. Hasenfratz and F. Knechtli, Phys. Rev. D64 (2001) 034504 [hep-lat/0103029]. 